\title{
Motion on Lie groups and its applications in Control Theory
}

\author{
José F. Cariñena \\ Departamento de Física Teórica, Facultad de Ciencias, \\ Universidad de Zaragoza, 50009 Zaragoza, Spain \\ e-mail: jfc@posta.unizar.es \\ Jesús Clemente-Gallardo \\ Instituto de Sistemas y Robotica, \\ Departamento de Engenharia Electrotecnica, \\ Universidade de Coimbra-Polo II, \\ Pinhal de Marrocos, 3030-290 Coimbra, Portugal \\ e-mail: jesus@isr.uc.pt \\ Arturo Ramos \\ Dipartimento di Matematica Pura ed Applicata, \\ Università degli Studi di Padova, \\ Via G. Belzoni 7, I-35131 Padova, Italy \\ e-mail: aramos@math.unipd.it
}




\begin{abstract}
The usefulness in control theory of the geometric theory of motion on Lie groups and homogeneous spaces will be shown. We quickly review some recent results concerning two methods to deal with these systems, namely, a generalization of the method proposed by Wei and Norman for linear systems, and a reduction procedure. This last method allows us to reduce the equation on a Lie group $G$ to that on a subgroup $H$, provided a particular solution of an associated problem in $G / H$ is known. These methods are shown to be very appropriate to deal with control systems on Lie groups and homogeneous spaces, through the specific examples of the planar rigid body with two oscillators and the front-wheel driven kinematic car.
\end{abstract}

Key words: Drift-free control systems, Wei-Norman method, motion in Lie groups and homogeneous spaces, reduction. 


\section{Introduction}

Mechanical systems whose configuration space is a Lie group are known to be of great relevance both in mechanics and control theory. The simplest example is that of a point particle moving freely on $\mathbb{R}^{3}$, for which the configuration space can be identified with the Abelian group of translations in a three-dimensional space. The motions of the rigid body are described by the Euclidean group in three dimensions, $E_{3}=T_{3} \odot S O(3, \mathbb{R})$, and for the rigid body with a fixed point the configuration space is $S O(3, \mathbb{R})$. However, there are also other systems which appear often in control theory which can be formulated in homogeneous spaces and reduced to problems of motion on Lie groups. The techniques used here have been developed in 2, 3, 4, 8, and firstly applied in control theory in [9, 10]. The paper is organized as follows. In the next section we review briefly the geometric theory of motion on Lie groups and homogeneous spaces. In Section 3 we recall two basic methods for dealing with such systems, and in Section 4 we illustrate the theory through its application on two control systems, namely, the planar rigid body with two oscillators and the front-wheel driven kinematic car.

\section{Motion on Lie groups and homogeneous spaces}

Given an $r$-dimensional connected Lie group $G$, the set of curves $\gamma: \mathbb{R} \rightarrow G$, $t \mapsto g(t)$, can be endowed with a group structure by means of the pointwise composition law, $\gamma_{2} * \gamma_{1}: t \mapsto g_{2}(t) g_{1}(t)$, for all $t \in \mathbb{R}$. Moreover, $G$ acts by right and left translations on itself, and if $g(t)$ defines a curve $\gamma$ starting from $g(0)=e$, then, for each $g_{0} \in G$, the maps $\gamma * \gamma_{0}: t \mapsto g(t) g_{0}$ and $\gamma_{0} * \gamma: t \mapsto g_{0} g(t)$ are two curves starting from $g_{0}$, which are called, respectively, the right and left translation of $\gamma$ by $g_{0}$.

In addition, the map $\dot{\gamma}: \mathbb{R} \rightarrow G$ given by $t \mapsto \dot{g}(t)$ defines a vector field along the curve $g(t)$, and then, using the right translation by $g^{-1}(t)$, we obtain a curve in $T_{e} G$ which can be written as

$$
\dot{g}(t) g^{-1}(t)=-\sum_{\alpha=1}^{r} b_{\alpha}(t) a_{\alpha},
$$

where $\left\{a_{1}, \ldots, a_{r}\right\}$ is a basis of the tangent space $T_{e} G$ at the neutral element $e \in G$. The minus sign on the right hand side is a matter of convention. 
The left hand side should be understood as $R_{g^{-1}(t) * g(t)}(\dot{g}(t))$, although for the particular case in which $G \subset G L(n, \mathbb{R})$, for certain $n$, it reduces to the above expression.

A first important point is that the curve $g(t)$ can be recovered as the only solution of equation (11) starting from $e \in G$. Any other solution is obtained from the previous $g(t)$ by right translation, and, in this sense, (II) is rightinvariant. In other words, $g(t)$ is the integral curve starting from $e \in G$ of the $t$-dependent vector field in $G$

$$
X(g, t)=-\sum_{\alpha=1}^{r} b_{\alpha}(t) X_{\alpha}^{R}(g)
$$

where $X_{\alpha}^{R}$ is the right-invariant vector field in $G$ whose value in $e \in G$ is $a_{\alpha}$, $X_{\alpha}^{R}(g)=R_{g * e}\left(a_{\alpha}\right)$. Similarly, $X_{\alpha}^{L}(g)=L_{g * e}\left(a_{\alpha}\right)$ denotes the left-invariant vector field on $G$ determined by $a_{\alpha}$. The left-invariant vector fields in $G$ close on a finite-dimensional Lie subalgebra $\mathfrak{g}$ of $\mathfrak{X}(G)$, which is called the Lie algebra of $G$. The right-invariant vector fields close on a finite-dimensional Lie algebra opposite to $\mathfrak{g}$.

Let us consider now a transitive action $\Phi: G \times M \rightarrow M$ of $G$ on a manifold $M$ (which is then called a homogeneous space of $G$ ). Each choice of a point $x_{0} \in M$ allows us to identify $M$ with a space of left cosets, i.e., $M=G / H$, where $H$ is the stability subgroup of the point $x_{0}$ with respect to $\Phi$. Different choices for $x_{0}$ lead to conjugate subgroups. We recall that $G$ can be regarded as the total space of the principal bundle $(G, \tau, G / H)$ over $G / H$, where $\tau: G \mapsto G / H$ is the canonical projection.

The important point now is 4] that the right-invariant vector fields $X_{\alpha}^{R}$ are $\tau$-projectable, the projections being the fundamental vector fields in $M$ associated to the natural left action of $G$ on $M=G / H$, identified with $\Phi$. More explicitly, we have $X_{\alpha}(x)=X_{a_{\alpha}}(x)=\Phi_{x * e}\left(-a_{\alpha}\right)$, where $x=g H$, and $\tau_{* g} X_{\alpha}^{R}(g)=-X_{\alpha}(g H)$. Consequently, the vector field (2) projects onto the $t$-dependent vector field in $M$

$$
X(x, t)=\sum_{\alpha=1}^{r} b_{\alpha}(t) X_{\alpha}(x),
$$

giving rise in this way to a system of differential equations

$$
\dot{x}^{i}=\sum_{\alpha=1}^{r} b_{\alpha}(t) X_{\alpha}^{i}(x), \quad i=1, \ldots, n=\operatorname{dim} M
$$


This relation between both systems tell us that the solution of (4) starting from the point $x_{0} \in M$, arbitrary but fixed, is given by $x(t)=\Phi\left(g(t), x_{0}\right)$, where $g(t)$ is the solution of (11) starting from the identity. Note that the vector fields arising in (4) close on the Lie algebra $\mathfrak{g}$.

Conversely, if a system of type (4) is defined by complete vector fields closing on a finite dimensional Lie algebra $\mathfrak{g}$, then it can be regarded as a system of the described type, taking a Lie group $G$ such that the vector fields are the fundamental vector fields of its action on the manifold $M$ given by the integration of their flows.

In this sense equation (11) has a universal character, since the knowledge of its solution starting from the identity is enough to write the general solution of each associated system of type (4) on each homogeneous space for $G$. Unfortunately, finding the desired particular solution of (1) may be a difficult problem. We briefly recall here two methods for dealing with such equations. The first one is based on a generalization [5, 8, of the method proposed by Wei and Norman for linear systems [17, 18]. The second one is a reduction procedure allowing us to reduce the problem to a similar one in a subgroup, when a solution of the associated problem in the corresponding homogeneous space is known [2, 3, 4, 8,

\section{The generalized Wei-Norman method and the reduction procedure}

The main idea of the generalization of the Wei-Norman method consists on writing the curve $g(t)$ solution of (11) starting from the identity, in terms of the second kind canonical coordinates with respect to a basis of the Lie algebra $\mathfrak{g},\left\{a_{1}, \ldots, a_{r}\right\}$, for all $t$, i.e., we write $g(t)=\prod_{\alpha=1}^{r} \exp \left(-v_{\alpha}(t) a_{\alpha}\right)$. Then, the differential equation (11) transforms into a system of differential equations for the $v_{\alpha}(t)$, and we have to find the solution determined by the initial conditions $v_{\alpha}(0)=0$ for all $\alpha=1, \ldots, r$. A simple calculation shows 8] that

$$
R_{g(t)^{-1} * g(t)}(\dot{g}(t))=-\sum_{\alpha=1}^{r} \dot{v}_{\alpha}\left(\prod_{\beta<\alpha} \exp \left(-v_{\beta}(t) \operatorname{ad}\left(a_{\beta}\right)\right)\right) a_{\alpha}
$$


and then, substituting into equation (II) we obtain the fundamental expression of the Wei-Norman method

$$
\sum_{\alpha=1}^{r} \dot{v}_{\alpha}\left(\prod_{\beta<\alpha} \exp \left(-v_{\beta}(t) \operatorname{ad}\left(a_{\beta}\right)\right)\right) a_{\alpha}=\sum_{\alpha=1}^{r} b_{\alpha}(t) a_{\alpha}
$$

with $v_{\alpha}(0)=0, \alpha=1, \ldots, r$. The resulting system of differential equations for the functions $v_{\alpha}(t)$ is integrable by quadratures if the Lie algebra is solvable [17, 18, and in particular, for nilpotent Lie algebras.

On the other hand, given an equation like (11) on a Lie group $G$, it may happen that the only non-vanishing coefficients $b_{\alpha}(t)$ are those corresponding to a subalgebra $\mathfrak{h}$ of $\mathfrak{g}$. In that case, the equation reduces to a simpler equation on a subgroup, involving less coordinates.

Now, the important result is that the general situation can be reduced to this simpler one under certain conditions. In fact, we can show that the problem of finding the solution of (11) starting at $e \in G$ can be reduced to the one of solving a similar equation in a subgroup $H$, provided that one particular solution $\tilde{g}_{1}(t)$ for the system defined by the left action $\Phi$ of $G$ on the homogeneous space $M=G / H$ is given. The result is as follows [4]:

Theorem 1 Every integral curve of the time-dependent vector field (2) in the group $G$ can be written in the form $g(t)=g_{1}(t) h(t)$, where $g_{1}(t)$ is a curve projecting onto a solution $\tilde{g}_{1}(t)$ of the system of type (4) associated to the left action $\Phi$ on the homogeneous space $G / H$, and $h(t)$ is a solution of an equation of type (1) but for the subgroup $H$, given explicitly by

$$
\left(\dot{h} h^{-1}\right)(t)=-\operatorname{Ad}\left(g_{1}^{-1}(t)\right)\left(\sum_{\alpha=1}^{r} b_{\alpha}(t) a_{\alpha}+\left(\dot{g}_{1} g_{1}^{-1}\right)(t)\right) \in T_{e} H .
$$

This result allows us to understand from a group theoretical point of view, as an example, the classical integration theorems for the Riccati equation involving one, two or three of its particular solutions [7], see also [4, 8].

\section{Some illustrative examples in control the- ory}

We will apply the previous techniques to problems appearing in the context of control theory, after a very brief description of such kind of problems. 
Roughly speaking, a control system is a dynamical system which depends on a set of control functions, $u_{\alpha}$, which model magnitudes to be varied somehow externally to the system, in order to modify its behaviour according to a specific purpose. For example, it could be desired to reach one point from another one of the configuration space, or to minimize some cost functional along the evolution of the system with prescribed initial and final conditions.

From a geometric point of view, we can describe the framework of a control system as a bundle $B$ (usually a trivial vector bundle) on the state space manifold $M$, with projection $\pi_{B}: B \rightarrow M$. The control dynamical system corresponds then to the integral curves of a vector field along the projection $\pi_{B}$, which in local coordinates, $\left(x^{i}, u_{\alpha}\right)$, reads

$$
\dot{x}^{i}=X^{i}(x, u), \quad x \in M, \quad u \in B_{x}=\pi_{B}^{-1}(x) .
$$

A special class is those of the control systems affine in the controls:

$$
\dot{x}^{i}=X_{0}^{i}(x)+\sum_{\alpha=1}^{r} u_{\alpha} X_{\alpha}^{i}(x), \quad i=1, \ldots, n=\operatorname{dim} M .
$$

The vector field $X_{0}^{i} \partial / \partial x^{i} \in \mathfrak{X}(M)$ is called the drift of the system. In principle, the control functions $u_{\alpha}$ are supposed to depend on time (in the control theory terminology the system is then operating in open loop).

We are now interested in those which are drift-less, i.e., $X_{0}=0$, since they become a system of type (4) as soon as the set of vector fields $\left\{X_{\alpha}^{i} \partial / \partial x^{i}\right\}$ closes on a finite-dimensional Lie algebra.

It may also happen that starting with a control system that is of the form (4), but where the vector fields $\left\{X_{\alpha}^{i} \partial / \partial x^{i}\right\}$ do not close on a Lie algebra, an appropriate feedback transformation $u_{\alpha}(x, t)=\sum_{\beta=1}^{r} f_{\alpha \beta}(x) v_{\beta}(t)$ could lead to a new system written as

$$
\dot{x}^{i}=\sum_{\alpha=1}^{r} v_{\alpha}(t) Y_{\alpha}^{i}(x), \quad i=1, \ldots, n=\operatorname{dim} M,
$$

where $Y_{\alpha}(x)=\sum_{i=1}^{r} f_{\beta \alpha}(x) X_{\beta}(x)$ do close a finite-dimensional Lie algebra.

We will illustrate by means of simple examples how systems of type (44), both directly or as a consequence of a feedback transformation, appear in control theory. In addition, with the general theory developed in previous sections, we will show how it is possible to solve, reduce, or relate systems of this kind formulated on different homogeneous spaces. 


\subsection{Planar rigid body with two oscillators}

This example comes from the consideration of the optimal control problem of a planar rigid body with two oscillators 19. The control system of interest has the state space $\mathbb{R}^{2} \times S^{1}$, with coordinates $\left(x_{1}, x_{2}, \theta\right)$

$$
\dot{x}_{1}=b_{1}(t), \quad \dot{x}_{2}=b_{2}(t), \quad \dot{\theta}=x_{1}^{2} b_{2}(t)-x_{2}^{2} b_{1}(t),
$$

where $b_{1}(t)$ and $b_{2}(t)$ are the control functions. This system is similar to the celebrated Brockett nonholonomic integrator system [1], but where the third equation is quadratic in the coordinates instead of linear, and the meaning of the third coordinate is now an angle.

The solutions of the system (10) are the integral curves of the timedependent vector field $b_{1}(t) X_{1}+b_{2}(t) X_{2}$, with

$$
X_{1}=\frac{\partial}{\partial x_{1}}-x_{2}^{2} \frac{\partial}{\partial \theta}, \quad X_{2}=\frac{\partial}{\partial x_{2}}+x_{1}^{2} \frac{\partial}{\partial \theta} .
$$

The Lie brackets

$$
X_{3}=\left[X_{1}, X_{2}\right]=2\left(x_{1}+x_{2}\right) \frac{\partial}{\partial \theta}, \quad X_{4}=\left[X_{1}, X_{3}\right]=2 \frac{\partial}{\partial \theta},
$$

jointly with $X_{1}, X_{2}$, make up a linearly independent set in points with $x_{1} \neq$ $-x_{2}$, and the set $\left\{X_{1}, X_{2}, X_{4}\right\}$ spans the tangent space at every point of $\mathbb{R}^{2} \times S^{1}$. According to Chow's theorem [1], every two such points can be joined by appropriate piecewise constant controls $b_{1}(t)$ and $b_{2}(t)$, therefore the system is controllable. In addition, the set $\left\{X_{1}, X_{2}, X_{3}, X_{4}\right\}$ closes on the nilpotent Lie algebra defined by the non-vanishing Lie brackets

$$
\left[X_{1}, X_{2}\right]=X_{3}, \quad\left[X_{1}, X_{3}\right]=X_{4}, \quad\left[X_{2}, X_{3}\right]=X_{4},
$$

isomorphic to a nilpotent Lie algebra, denoted as $\mathfrak{g}_{4}$, which can be regarded as a central extension of the Heisenberg Lie algebra $\mathfrak{h}(3)$ by $\mathbb{R}$. In fact, taking the basis $\left\{a_{1}, a_{2}, a_{3}, a_{4}\right\}$ of $\mathfrak{g}_{4}$ for which the non-vanishing Lie products are

$$
\left[a_{1}, a_{2}\right]=a_{3}, \quad\left[a_{1}, a_{3}\right]=a_{4}, \quad\left[a_{2}, a_{3}\right]=a_{4},
$$

then the center $\mathfrak{z}$ of the algebra is generated by $\left\{a_{4}\right\}$, and the factor Lie algebra $\mathfrak{g}_{4} / \mathfrak{z}$ is isomorphic to the Heisenberg Lie algebra $\mathfrak{h}(3)$. 
Let $G_{4}$ be the connected and simply connected nilpotent Lie group such that its Lie algebra is the previous $\mathfrak{g}_{4}$. The right-invariant system of type (II) on $G_{4}$ corresponding to (10) is

$$
R_{g(t)^{-1} * g(t)}(\dot{g}(t))=-b_{1}(t) a_{1}-b_{2}(t) a_{2} .
$$

Let us solve it by the Wei-Norman method. We write the solution of (14) starting from the identity as the product of exponentials

$$
g(t)=\exp \left(-v_{1}(t) a_{1}\right) \exp \left(-v_{2}(t) a_{2}\right) \exp \left(-v_{3}(t) a_{3}\right) \exp \left(-v_{4}(t) a_{4}\right)
$$

and using the expression of the adjoint representation of $\mathfrak{g}_{4}$, and its exponentiation, and applying then (5), we find the system

$$
\dot{v}_{1}=b_{1}(t), \quad \dot{v}_{2}=b_{2}(t), \quad \dot{v}_{3}=b_{2}(t) v_{1}, \quad \dot{v}_{4}=b_{2}(t) v_{1}\left(v_{1} / 2+v_{2}\right),
$$

with initial conditions $v_{1}(0)=v_{2}(0)=v_{3}(0)=v_{4}(0)=0$. The solution is easily found by quadratures: if we denote $B_{i}(t)=\int_{0}^{t} b_{i}(s) d s, i=1,2$, then,

$$
\begin{aligned}
& v_{1}(t)=B_{1}(t), \quad v_{2}(t)=B_{2}(t), \quad v_{3}(t)=\int_{0}^{t} b_{2}(s) B_{1}(s) d s . \\
& v_{4}(t)=\int_{0}^{t} b_{2}(s)\left(\frac{1}{2} B_{1}^{2}(s)+B_{1}(s) B_{2}(s)\right) d s .
\end{aligned}
$$

Other orderings in the factorization (15) are possible, with similar results.

Now, we can find the expressions of the action $\Phi$ of $G_{4}$ on the configuration manifold $\mathbb{R}^{2} \times S^{1}$ such that $X_{i}$ be the infinitesimal generator associated to $a_{i}$ for each $i \in\{1, \ldots, 4\}$, and of the composition law of $G_{4}$. We will use canonical coordinates of the second kind in $G_{4}$ defined by $g=\exp \left(a a_{1}\right) \exp \left(b a_{2}\right) \exp \left(c a_{3}\right) \exp \left(d a_{4}\right)$, and then, integrating and composing accordingly the flows of the vector fields $X_{i}$, the action reads $\Phi$ : $G_{4} \times\left(\mathbb{R}^{2} \times S^{1}\right) \rightarrow \mathbb{R}^{2} \times S^{1}$

$$
\begin{aligned}
\Phi\left((a, b, c, d),\left(x_{1}, x_{2}, \theta\right)\right) & =\left(x_{1}-a, x_{2}-b,\right. \\
\theta & \left.+a x_{2}^{2}-b x_{1}^{2}-2(a b+c) x_{2}-2 c x_{1}+a b^{2}-2 d\right),
\end{aligned}
$$

and the composition law of $G_{4}$ reads

$$
\begin{aligned}
&(a, b, c, d)\left(a^{\prime}, b^{\prime}, c^{\prime}, d^{\prime}\right)=\left(a+a^{\prime}, b+b^{\prime}, c+c^{\prime}-b a^{\prime},\right. \\
&\left.d+d^{\prime}-c\left(a^{\prime}+b^{\prime}\right)+b a^{\prime}\left(b+2 b^{\prime}+a^{\prime}\right) / 2\right) .
\end{aligned}
$$


The neutral element is represented by $(0,0,0,0)$ in these coordinates.

The general solution of (10) can be calculated by means of the solution of the Wei-Norman system (16) as

$$
\begin{aligned}
& \Phi\left(\left(-v_{1},-v_{2},-v_{3},-v_{4}\right),\left(x_{10}, x_{20}, \theta_{0}\right)\right)=\left(x_{10}+v_{1}, x_{20}+v_{2},\right. \\
& \left.\theta_{0}-v_{1} x_{20}^{2}+v_{2} x_{10}^{2}-2\left(v_{1} v_{2}-v_{3}\right) x_{20}+2 v_{3} x_{10}-v_{1} v_{2}^{2}+2 v_{4}\right),
\end{aligned}
$$

where $v_{1}=v_{1}(t), v_{2}=v_{2}(t), v_{3}=v_{3}(t)$ and $v_{4}=v_{4}(t)$ are given by (17), $\left(x_{10}, x_{20}, \theta_{0}\right) \in \mathbb{R}^{2} \times S^{1}$ are the initial conditions and $\Phi$ is given by (18).

We will show the way the previous reduction theorem applies to the control system (10). Several possibilities of reduction exist, as many as (nonequivalent) subgroups of $G_{4}$. Specifically, we will show how the system (14) can be reduced to a control system of Brockett type plus a system on the real line, performing the reduction with respect to the center of $G_{4}$.

For a better illustration we parametrize now $G_{4}$ by canonical coordinates of first kind defined by $g=\exp \left(a a_{1}+b a_{2}+c a_{3}+d a_{4}\right)$, being then the composition law

$$
\begin{aligned}
& (a, b, c, d)\left(a^{\prime}, b^{\prime}, c^{\prime}, d^{\prime}\right)=\left(a+a^{\prime}, b+b^{\prime}, c+c^{\prime}+\left(a b^{\prime}-b a^{\prime}\right) / 2,\right. \\
& \left.d+d^{\prime}+\left(a c^{\prime}-c a^{\prime}\right) / 2+\left(b c^{\prime}-c b^{\prime}\right) / 2+\left(a b^{\prime}-b a^{\prime}\right)\left(a-a^{\prime}+b-b^{\prime}\right) / 12\right) .
\end{aligned}
$$

Thus, the adjoint representation of the group reads

$$
\operatorname{Ad}(a, b, c, d)=\left(\begin{array}{cccc}
1 & 0 & 0 & 0 \\
0 & 1 & 0 & 0 \\
-b & a & 1 & 0 \\
-\frac{b}{2}(a+b)-c & \frac{a}{2}(a+b)-c & a+b & 1
\end{array}\right)
$$

If $g(t)=(a(t), b(t), c(t), d(t))$ is a curve in $G_{4}$ expressed in the previous coordinates, we obtain

$$
R_{g^{-1} * g}(\dot{g})=\left(\begin{array}{c}
\dot{a} \\
\dot{b} \\
\dot{c}-\frac{1}{2}(b \dot{a}-a \dot{b}) \\
\dot{d}-\frac{1}{6}\left(a b+b^{2}+3 c\right) \dot{a}+\frac{1}{6}\left(a^{2}+a b-3 c\right) \dot{b}+\frac{1}{2}(a+b) \dot{c}
\end{array}\right) .
$$

To perform the reduction we select the subgroup $H$ to be the center of $G_{4}$, which is generated by $a_{4}$. The relevant factorization is $(a, b, c, d)=$ 
$(a, b, c, 0)(0,0,0, d)$. Therefore, we can describe the homogeneous space $M=G_{4} / H$ by means of the projection

$$
\begin{aligned}
\tau: G_{4} & \longrightarrow G_{4} / H \\
(a, b, c, d) & \longmapsto(a, b, c),
\end{aligned}
$$

associated to the previous factorization. We take coordinates $\left(y_{1}, y_{2}, y_{3}\right)$ in $M$. Thus, the left action of $G_{4}$ on such a homogeneous space reads

$$
\begin{aligned}
\Phi: G_{4} \times M & \longrightarrow M \\
\left((a, b, c, d),\left(y_{1}, y_{2}, y_{3}\right)\right) & \longmapsto\left(y_{1}+a, y_{2}+b, y_{3}+c+\left(a y_{2}-b y_{1}\right) / 2\right) .
\end{aligned}
$$

The corresponding fundamental vector fields are

$$
X_{1}^{H}=-\frac{\partial}{\partial y_{1}}-\frac{y_{2}}{2} \frac{\partial}{\partial y_{3}}, \quad X_{2}^{H}=-\frac{\partial}{\partial y_{2}}+\frac{y_{1}}{2} \frac{\partial}{\partial y_{3}}, \quad X_{3}^{H}=-\frac{\partial}{\partial y_{3}}, \quad X_{4}^{H}=0,
$$

which span the tangent space at each point of $M$, and, in addition, satisfy the Lie brackets $\left[X_{1}^{H}, X_{2}^{H}\right]=X_{3}^{H},\left[X_{1}^{H}, X_{3}^{H}\right]=X_{4}^{H}=0$ and $\left[X_{2}^{H}, X_{3}^{H}\right]=$ $X_{4}^{H}=0$.

Now, let the desired solution of (14) be factorized as the product

$$
g_{1}(t) h(t)=\left(y_{1}(t), y_{2}(t), y_{3}(t), 0\right)(0,0,0, d(t))
$$

where $g_{1}(t)$ projects onto the solution $\tau\left(g_{1}(t)\right)=\left(y_{1}(t), y_{2}(t), y_{3}(t)\right)$, with initial conditions $(0,0,0)$, of the Lie system on the homogeneous space $M$ associated to (14),

$$
\dot{y}_{1}=-b_{1}(t), \quad \dot{y}_{2}=-b_{2}(t), \quad \dot{y}_{3}=\frac{1}{2}\left(b_{2}(t) y_{1}-b_{1}(t) y_{2}\right) .
$$

Then, we reduce the problem to a Lie system in the subgroup $H$ for $h(t)=$ $(0,0,0, d(t))$, with $h(0)=e$, i.e., $d(0)=0$. The expression of this last system is given by Theorem 11, i.e.,

$$
\dot{d}=\frac{1}{12}\left(\left(y_{1}+y_{2}\right)\left(b_{1} y_{2}-b_{2} y_{1}\right)-6 y_{3}\left(b_{1}+b_{2}\right)\right)
$$

which is a system on $H \cong \mathbb{R}$, solvable by one quadrature. The system (20) is of Brockett type [1] (indeed they are related by the simple change of coordinates $x=-y_{1}, y=-y_{2}$ and $z=-2 y_{3}$ ), and therefore we obtain two 
interesting results. Firstly, that solving a system of type (10) can be reduced to solving first a system of Brockett type and then to solving a Lie system in $\mathbb{R}$, which is immediate. Secondly, that the Brockett system can be regarded as a Lie system on $H(3)$ written, moreover, in terms of canonical coordinates of first kind of such a group.

As an interesting open problem, it remains to investigate the interrelations the corresponding optimal control problems might have with respect to this reduction.

\subsection{Front-wheel driven kinematic car}

The front-wheel driven kinematic car has been considered by a number of authors, mainly with regard to the nonholonomic motion planning problem, and as such is made nilpotent by a state space feedback transformation [12, 13, 14, 15, 16. It is a simple model of a car with front and rear wheels. The distance between the rear and front axles is $l$, which we will take as 1 for simplicity.

The configuration of the car is determined by the Cartesian coordinates $(x, y)$ of the rear wheels, the angle of the car body $\theta$ with respect to the horizontal coordinate axis, and the steering front wheel angle $\phi \in I=$ $(-\pi / 2, \pi / 2)$ relative to the car body. The configuration space is therefore $\mathbb{R}^{2} \times S^{1} \times I$, with coordinates $(x, y, \theta, \phi)$. The controls of the system are the velocity of the rear (or sometimes front) wheels and the turning speed of the front wheels. For a schematic picture of the system, see, e.g., [14, 16].

The control system for this model can be written as [14, 16] (compare with [12, Eq. (13.7)])

$$
\dot{x}=c_{1}(t), \quad \dot{y}=c_{1}(t) \tan \theta, \quad \dot{\phi}=c_{2}(t), \quad \dot{\theta}=c_{1}(t) \tan \phi \sec \theta .
$$

Note that this system is defined for angles $\theta$ with $\cos \theta \neq 0$. We therefore restrict $\theta \in I$ as well. The solutions of (21) are the integral curves of the time-dependent vector field $c_{1}(t) Y_{1}+c_{2}(t) Y_{2}$, where

$$
Y_{1}=\frac{\partial}{\partial x}+\tan \theta \frac{\partial}{\partial y}+\tan \phi \sec \theta \frac{\partial}{\partial \theta}, \quad Y_{2}=\frac{\partial}{\partial \phi}
$$

Taking the Lie brackets

$$
Y_{3}=\left[Y_{1}, Y_{2}\right]=-\sec \theta \sec ^{2} \phi \frac{\partial}{\partial \theta}, \quad Y_{4}=\left[Y_{1}, Y_{3}\right]=\sec ^{2} \theta \sec ^{2} \phi \frac{\partial}{\partial y}
$$


we see that $\left\{Y_{1}, Y_{2}, Y_{3}, Y_{4}\right\}$ generate the full tangent space at points of the (restricted) configuration space $\mathbb{R}^{2} \times I \times I$, thus the system is controllable there. However, (21) is not a system of type (41), since the iterated Lie brackets

$$
\left[Y_{2},\left[Y_{2}, \ldots\left[Y_{2}, Y_{1}\right] \cdots\right]\right] \quad \text { or } \quad\left[Y_{1},\left[Y_{1}, \ldots\left[Y_{1}, Y_{2}\right] \cdots\right]\right]
$$

generate at each step vector fields linearly independent from those obtained at the previous stage, therefore they do not close a finite-dimensional Lie algebra.

Notwithstanding, it can be transformed into a nilpotent system of type (41). In [12, 14, 16] it is proposed the following state space feedback transformation (it seems that in [14, 16] there are some minor misprints, for their expressions do not do the work)

$$
c_{1}(t)=b_{1}(t) \quad c_{2}(t)=-3 \sin ^{2} \phi \sec ^{2} \theta \sin \theta b_{1}(t)+\cos ^{3} \theta \cos ^{2} \phi b_{2}(t),
$$

and then the change of coordinates

$$
x_{1}=x, \quad x_{2}=\sec ^{3} \theta \tan \phi, \quad x_{3}=\tan \theta, \quad x_{4}=y,
$$

with inverse

$$
x=x_{1}, \quad y=x_{4}, \quad \theta=\arctan x_{3}, \quad \phi=-\arctan \left(\frac{x_{2}}{\left(1+x_{3}^{2}\right)^{3 / 2}}\right),
$$

which transforms (21) into the control system in $\mathbb{R}^{4}$ with coordinates $\left(x_{1}, x_{2}, x_{3}, x_{4}\right)$ given by

$$
\dot{x}_{1}=b_{1}(t), \quad \dot{x}_{2}=b_{2}(t), \quad \dot{x}_{3}=b_{1}(t) x_{2}, \quad \dot{x}_{4}=b_{1}(t) x_{3},
$$

where the control functions are now $b_{1}(t)$ and $b_{2}(t)$. In other words, we take the new input vector fields

$$
X_{1}=Y_{1}-3 \sin ^{2} \phi \sec ^{2} \theta \sin \theta Y_{2}, \quad X_{2}=\cos ^{3} \theta \cos ^{2} \phi Y_{2} .
$$

The system (25) is usually said to be in chained form, see [14, 16]. Let us show that it is a system of type (4). The new input vector fields read, in the coordinates $\left(x_{1}, x_{2}, x_{3}, x_{4}\right)$, as

$$
X_{1}=\frac{\partial}{\partial x_{1}}+x_{2} \frac{\partial}{\partial x_{3}}+x_{3} \frac{\partial}{\partial x_{4}}, \quad X_{2}=\frac{\partial}{\partial x_{2}} .
$$


The Lie brackets

$$
X_{3}=\left[X_{1}, X_{2}\right]=-\frac{\partial}{\partial x_{3}}, \quad X_{4}=\left[X_{1}, X_{3}\right]=\frac{\partial}{\partial x_{4}},
$$

are linearly independent from $X_{1}$ and $X_{2}$, and $\left\{X_{1}, X_{2}, X_{3}, X_{4}\right\}$ generate the full tangent space at every point of the configuration space $\mathbb{R}^{4}$, so the system is controllable. On the other hand, the same set closes on the nilpotent Lie algebra defined by the non-vanishing Lie brackets

$$
\left[X_{1}, X_{2}\right]=X_{3}, \quad\left[X_{1}, X_{3}\right]=X_{4}
$$

This Lie algebra is isomorphic to a four dimensional nilpotent Lie algebra, denoted by $\overline{\mathfrak{g}}_{4}$, which is also a central extension of the Lie algebra $\mathfrak{h}(3)$ by $\mathbb{R}$. Indeed, taking the basis $\left\{a_{1}, a_{2}, a_{3}, a_{4}\right\}$ of $\overline{\mathfrak{g}}_{4}$ such that the non-vanishing defining relations are

$$
\left[a_{1}, a_{2}\right]=a_{3}, \quad\left[a_{1}, a_{3}\right]=a_{4},
$$

then the center $\mathfrak{z}$ of the algebra is generated by $\left\{a_{4}\right\}$, and the factor Lie algebra $\overline{\mathfrak{g}}_{4} / \mathfrak{z}$ is isomorphic to $\mathfrak{h}(3)$. However, this extension is not equivalent to the extension appearing in the case of the planar rigid body with two oscillators, compare (28) with (13).

We give now briefly some results concerning the solution of (25) by the Wei-Norman method and its reduction to a system of Brockett type plus a system in $\mathbb{R}$. The calculations are similar to that of the previous subsection.

Let $\bar{G}_{4}$ be the connected and simply connected nilpotent Lie group whose Lie algebra is $\overline{\mathfrak{g}}_{4}$. The right-invariant system of type (11) on $\bar{G}_{4}$ corresponding to (25) reads as (14) but where now $g(t)$ is the solution curve in $\bar{G}_{4}$ starting from the identity, and $\left\{a_{1}, a_{2}, a_{3}, a_{4}\right\}$ is the previous basis of $\overline{\mathfrak{g}}_{4}$. Writing $g(t)=\exp \left(-v_{1} a_{1}\right) \exp \left(-v_{2} a_{2}\right) \exp \left(-v_{3} a_{3}\right) \exp \left(-v_{4} a_{4}\right)$, the Wei-Norman method gives the system

$$
\dot{v}_{1}=b_{1}(t), \quad \dot{v}_{2}=b_{2}(t), \quad \dot{v}_{3}=b_{2}(t) v_{1}, \quad \dot{v}_{4}=b_{2}(t) \frac{v_{1}^{2}}{2}
$$

with initial conditions $v_{1}(0)=v_{2}(0)=v_{3}(0)=v_{4}(0)=0$, which is easily integrable by quadratures. The action $\Phi$ of $\bar{G}_{4}$ on $\mathbb{R}^{4}$ corresponding to the infinitesimal generators $\left\{X_{i}\right\}$ reads, parametrizing $g \in \bar{G}_{4}$ by $g=$ $\exp \left(a a_{1}\right) \exp \left(b a_{2}\right) \exp \left(c a_{3}\right) \exp \left(d a_{4}\right)$,

$$
\begin{aligned}
\Phi: \bar{G}_{4} \times \mathbb{R}^{4} & \longrightarrow \mathbb{R}^{4} \\
\left((a, b, c, d),\left(x_{1}, x_{2}, x_{3}, x_{4}\right)\right) & \longmapsto\left(\bar{x}_{1}, \bar{x}_{2}, \bar{x}_{3}, \bar{x}_{4}\right),
\end{aligned}
$$


where $\bar{x}_{1}=x_{1}-a, \bar{x}_{2}=x_{2}-b, \bar{x}_{3}=x_{3}-a x_{2}+a b+c$ and $\bar{x}_{4}=x_{4}-a x_{3}+$ $a^{2} x_{2} / 2-a^{2} b / 2-a c-d$. The composition law reads

$$
(a, b, c, d)\left(a^{\prime}, b^{\prime}, c^{\prime}, d^{\prime}\right)=\left(a+a^{\prime}, b+b^{\prime}, c+c^{\prime}-b a^{\prime}, d+d^{\prime}-c a^{\prime}+b a^{2} / 2\right),
$$

the neutral element being represented by $(0,0,0,0)$.

Thus, the general solution of (25) is

$$
\begin{aligned}
& \Phi\left(\left(-v_{1},-v_{2},-v_{3},-v_{4}\right),\left(x_{10}, x_{20}, x_{30}, x_{40}\right)\right)=\left(x_{10}+v_{1}, x_{20}+v_{2},\right. \\
& \left.x_{30}+v_{1} x_{20}+v_{1} v_{2}-v_{3}, x_{40}+v_{1} x_{30}+v_{1}^{2} x_{20} / 2+v_{1}^{2} v_{2} / 2-v_{1} v_{3}+v_{4}\right),
\end{aligned}
$$

where $v_{1}=v_{1}(t), v_{2}=v_{2}(t), v_{3}=v_{3}(t)$ and $v_{4}=v_{4}(t)$ are the solution of (29) and the initial conditions are $\left(x_{10}, x_{20}, x_{30}, x_{40}\right) \in \mathbb{R}^{4}$.

Due to the Lie algebra structure of $\overline{\mathfrak{g}}_{4}$, by quotienting by the center, we can reduce the solution of the equation in the group $\bar{G}_{4}$ (and hence of (25)) to two other problems: one, a system in $H(3)$ which is of Brockett type, and then we have to integrate a system in $\mathbb{R}$. Taking canonical coordinates of first kind, and following analogous steps to that of the previous subsection, we have to solve first (20), and then

$$
\dot{d}=\frac{b_{1}(t)}{2}\left(\frac{1}{6} y_{1}(t) y_{2}(t)-y_{3}(t)\right)-\frac{1}{12} b_{2}(t) y_{1}^{2}(t)
$$

which is integrable by one quadrature.

\section{Acknowledgements}

Partial support of the Spanish DGI, project BFM2000-1066-C03-01, is acknowledged. A.R. has been partially supported by the Spanish Ministerio de Ciencia y Tecnología through a FPI grant and by the European Commission funding for the Human Potential Research Network "Mechanics and Symmetry in Europe" (MASIE), contract HPRN-CT-2000-00113. J.C.-G. has been partially supported by the European Union through the TMR Network in Nonlinear Control (Contract ERB FMRXCT-970137). 


\section{References}

[1] R.W. Brockett: Control theory and singular Riemannian geometry, in: New directions in applied mathematics, P.J. Hilton and G.S. Young Eds., Springer, New York 1982.

[2] J.F. Cariñena, J. Grabowski and G. Marmo: Lie-Scheffers systems: a geometric approach, Bibliopolis, Napoli 2000.

[3] J.F. Cariñena, J. Grabowski and G. Marmo: Rep. Math. Phys. 48 (2001), 47-58.

[4] J.F. Cariñena, J. Grabowski and A. Ramos: Acta Appl. Math. 66 (2001), $67-87$.

[5] J.F. Cariñena, G. Marmo and J. Nasarre: Int. J. Mod. Phys. A 13 (1998), 3601-3627.

[6] J.F. Cariñena and J. Nasarre: J. Opt. B: Quantum Semiclass. Opt. 2 (2000), 94-99.

[7] J.F. Cariñena and A. Ramos, Int. J. Mod. Phys. A 14 (1999), 19351951.

[8] J.F. Cariñena and A. Ramos: Acta Appl. Math. 70 (2002), 43-69.

[9] J.F. Cariñena and A. Ramos: Lie systems in control theory, in: Contemporary trends in nonlinear geometric control theory and its applications, pp. 287-304, A. Anzaldo-Meneses, B. Bonnard, J.-P. Gauthier and F. Monroy-Pérez Eds., World Scientific, Singapore 2002.

[10] J.F. Cariñena and A. Ramos: Applications of Lie systems in quantum mechanics and control theory, in: Classical and Quantum Integrability, p. 143-62, Banach Center Publications v. 59, 2003.

[11] W.-L. Chow: Math. Ann. 117 (1940/1941), 98-105.

[12] G. Lafferriere and H.J. Sussmann: Motion planning for controllable systems without drift, in Proc. IEEE Conf. Robotics and Automation, IEEE Publications, New York 1991. 
[13] G. Lafferriere and H.J. Sussmann: A differential geometric approach to motion planning, in Nonholonomic motion planning, Z.X. Li and J.F. Canny Eds., Kluwer, Norwell 1993.

[14] R.M. Murray: Control of nonholonomic systems using chained form, in: Dynamics and control of mechanical systems, the falling cat and related problems, M.J. Enos Ed., Fields Institute Communications 1, Amer. Math. Soc., Providence, Rhode Island 1993.

[15] R.M. Murray and S.S. Sastry: Steering nonholonomic systems in chained form, in: Proc. IEEE Conf. Decision and Control, IEEE Publications, New York 1991.

[16] R.M. Murray and S.S. Sastry: IEEE T. Automat. Contr. 38 (1993), $700-716$.

[17] J. Wei and E. Norman: J. Math. Phys. 4 (1963), 575-581.

[18] J. Wei and E. Norman: Proc. Amer. Math. Soc. 15 (1964), 327-334.

[19] R. Yang, P.S. Krishnaprasad and W. Dayawansa: Optimal control of a rigid body with two oscillators, in: Mechanics day, Fields Institute Communications 7, Amer. Math. Soc., Providence, Rhode Island 1996. 